\title{
The challenges on developing vaccine against visceral leishmaniasis
}

\author{
Selma Maria Bezerra Jerônimo ${ }^{[1],[2],[3]}$ and Richard D. Pearson ${ }^{[4]}$
}

\begin{abstract}
[1]. Departamento de Bioquímica, Centro de Biociências, Universidade Federal do Rio Grande do Norte, Natal, Rio Grande do Norte, Brasil. [2]. Departamento de Bioquímica, Universidade Federal do Rio Grande do Norte, Natal, Rio Grande do Norte, Brasil.

[3] Instituto Nacional de Ciências e Tecnologia de Doenças Tropicais, INCT-DT, Salvador, Bahia, Brasil.

[4] Departments of Internal Medicine and Pathology, University of Virginia, Charlottesville, VA, USA.
\end{abstract}

Leishmaniasis is a complex of diseases caused by species of Leishmania. The outcome of Leishmania infection ranges from asymptomatic, self-resolving infection to cutaneous, mucosal, disseminated or visceral disease ${ }^{(1)}$. The risk of developing clinically apparent disease depends in part on the infecting Leishmania species, the environmental, host susceptibility factors, and co-morbidities ${ }^{(2)}$. For instance, Leishmania donovani typically causes visceral leishmaniasis, but occasionally can present as cutaneous disease only; Leishmania braziliensis can cause cutaneous, mucosal or disseminated disease, whereas Leishmania amazonensis can cause cutaneous, diffuse or visceral leishmaniasis. Together, leishmaniasis continues to plague many areas of the world and new epidemiological trends have been seen with migration, military conflicts, and human immunodeficiency virus (HIV) pandemic.

Visceral leishmaniasis can be fatal in 5 to $10 \%$ of the cases even with treatment ${ }^{(1)}$, whereas the other forms of leishmaniasis can evolve with high morbidity. People suffering from mucosal leishmaniasis can present with severe disfigurement. There is only a small repertoire of drugs available that are effective in the treatment of leishmaniasis. Pentavalent antimonials have been a mainstay of treatment for decades, but toxicity and increased resistance have led to their decreased use in most areas of the world. Liposomal amphotericin has become accessible in many endemic countries and is approved for visceral leishmaniasis by the United States Food and Drug Administration (FDA), as is miltefosine, the first oral medication for leishmaniasis. However, treatment failures and resistance have been reported with those drugs as well ${ }^{(1)}$. Failure can also be a consequence of delay in therapy or due to co-morbidities that affect immune responses. A vaccine against leishmaniasis is needed to protect vulnerable populations.

Corresponding author: Dra. Selma M.B. Jeronimo.

e-mail: selma.b.jeronimo@gmail.com

Received 25 August 2016

Accepted 26 August 2016
While no effective human vaccine against cutaneous, mucosal or visceral human leishmaniasis is available ${ }^{(3)}$, vaccines against canine visceral leishmaniasis due to $L$. infantum have been used in Europe and Brazil ${ }^{(4)}$. Data on the long term efficacy are still being collected. An effective vaccine against canine leishmaniasis could have important implications for human visceral leishmaniasis in regions where domestic dogs are the major reservoir, but will not be relevant in areas where rodents or humans are major reservoir of infection.

In this issue of the Revista da Sociedade Brasileira de Medicina Tropical, Duarte MC et $\mathrm{al}^{(4)}$ present updates and perspectives on different approaches that have been used to date in development of vaccines against leishmaniasis. First, they review the data on second generation vaccines, which are composed mainly of single recombinant antigens derived from amastigotes or promastigotes that have provided partial or high level protection. The protein A2 has emerged as a potentially effective candidate providing protective immunity and generation of immunoglobulin $\mathrm{G} 2 \mathrm{a}$ (IgG2a) antibodies and high levels of interferon gamma (IFN-y). Other recombinant proteins such as cysteine proteinases, parasite surface antigen-2, and kinetoplastid membrane protein-11 (KMP-11) have been shown to have effect in various animal models ${ }^{(4)}$.

The next approach discussed is the use of chimeric antigens. During formulation, proteins expressed by both forms of Leishmania, promastigote and amastigote, and with multiple epitopes have been used to increase the likelihood of a protective response. Polyproteins such as KSAC, with sequences from the Leishmania homolog of the receptor for activated C kinase (LACK), glycoprotein $63 \mathrm{kDa}$ (gp63), thiol-specific-antioxidant, hydrophilic acylated surface protein B, sterol 24-c-metlhytransferase, KMP-11, A2 and cysteine proteinase $\mathrm{B}(\mathrm{CPB})$ proteins, have been formulated with adjuvants as monophosphoryl lipid A, and shown promising results. Finally, newly identified Leishmania antigens have been selected based on their recognition by sera from symptomatic and asymptomatic dogs using immune proteomic platforms $\mathrm{s}^{(5)}$. These antigens have been sequenced and then analyzed in silico resulting in identification of specific cluster of differentiation 4+ 
$(\mathrm{CD} 4+)$ and cluster of differentiation $8+(\mathrm{CD} 8+)$ epitopes. The hope is that these antigens will confer protection for multiple Leishmania species, and several candidates are in the process of being tested ${ }^{(4)}$. The concept of formulating cross-protective vaccines based on common antigens in the Leishmania genus and their ability to elicit a particular type of immune response is interesting, but also a challenge. Although partial protection against $L$. infantum infection has been observed when a Leishmania braziliensis hypothetical protein and eukaryotic initiation factor 5a (EiF5a) were used in conjunction as a polyprotein vaccine in a mouse $\operatorname{model}^{(5)}$, this has yet to be shown in other animals.

It is well recognized that the goal in reaching protection against visceralizing Leishmania species is to overcome the immunosuppression that occurs with progression to disease. (1) In contrast the immune response may need to be dampened in order to decrease pathology in tegumentary leishmaniasis ${ }^{(6)}$. Overall, protection against Leishmania species that cause visceral leishmaniasis depends on the development of a strong T-helper 1 (Th1) type of response with concomitant CD8+ responses (1), whereas in tegumentary leishmaniasis, such as the one seen in Leishmania braziliensis infection, a strengthened immune response is necessary to control the parasite burden, including an increased CD8+ response, but is also associated with pathology $y^{(6)}$. Therefore, attention to the immune responses elicited by vaccine candidates needs to be carefully considered in light of the balance between the need to control parasite replication and the responses involved in pathology.

Studies of symptomatic and asymptomatic infections due to either $L$. donovani or $L$. infantum can be of help in designing strategies for vaccine development. Because both asymptomatic and symptomatic infections can result in long-lasting protective immunity against Leishmania, an effective vaccine may be attainable. In endemic areas, the majority of infected people naturally have self-resolving infections. Figure 1 illustrates potential outcomes associated with $L$. infantum infection in humans; both symptomatic and asymptomatic people eventually mount a strong delayed-type hypersensitivity (DTH) response, which is a proxy for effective CD4+ producing IFN-y. However, the outcome of infection can be skewed to disease. There is an increased risk of developing symptomatic visceral leishmaniasis if immunosuppression occurs because of a neoplastic disorder ${ }^{(7)}$, $\mathrm{HIV}^{(8)}$, or malnutrition ${ }^{(9)}$. Risk of disease is also associated to host genetics ${ }^{(10)}$. All of those factors need to be examined during vaccine design.

Another consideration in vaccine development is the role of vector salivary antigens ${ }^{(4)}$. Components of sand fly salivary glands drive potent Th1 immune responses in immunized

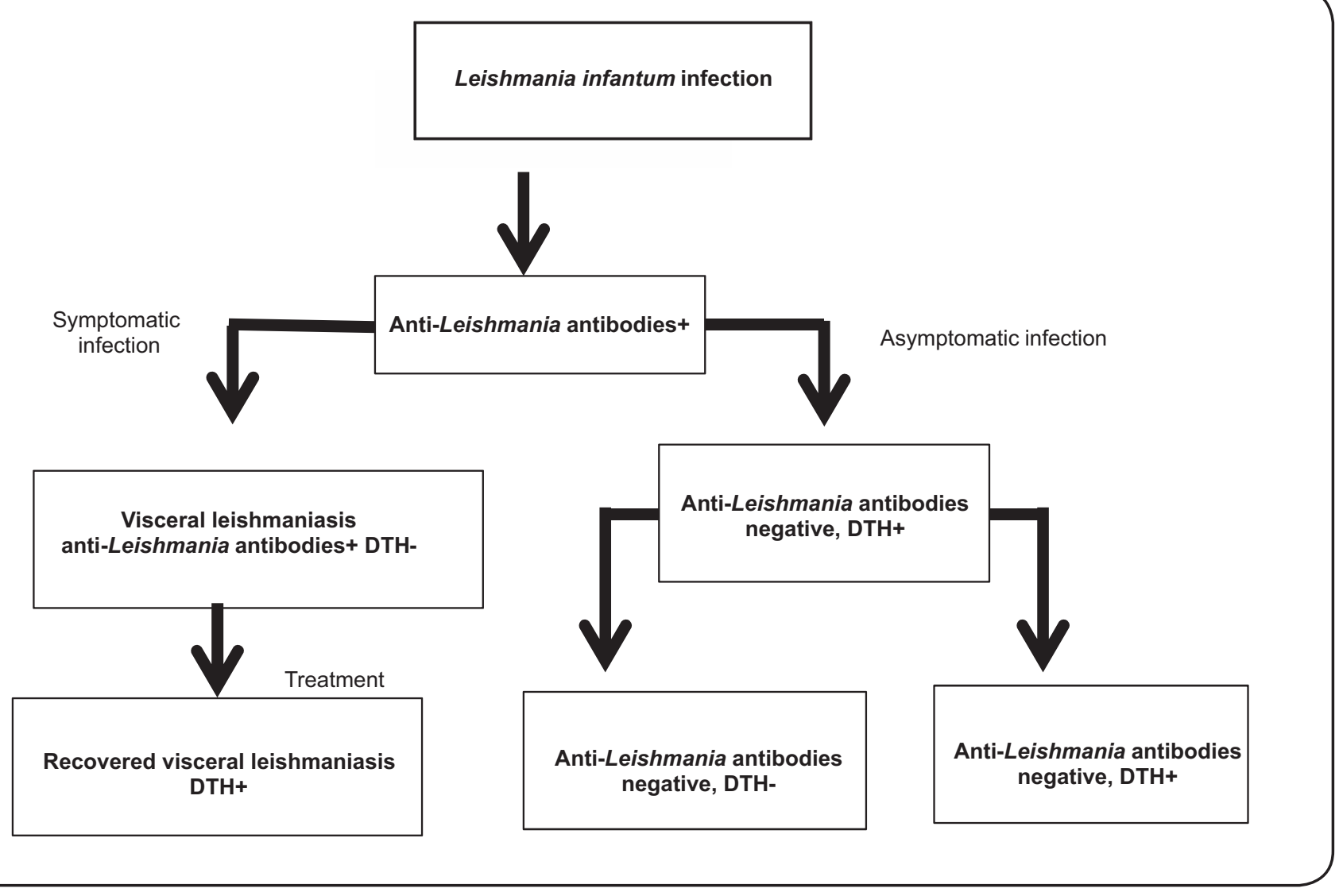

FIGURE 1. Outcomes of Leishmania infantum in humans. The majority of people infected with Leishmania infantum develop a protective response which is long lasting. DTH: delayed-type hypersensitivity. 
mice, but also in people naturally exposed to sand flies. These antigens could elicit a protective immune response at the bite site where promastigotes are inoculated by infected sand flies, where these pathogens are potentially more vulnerable and a better target for the human immune system ${ }^{(3)}$. Therefore, it has been proposed that a model vaccine against leishmaniasis should include sand fly salivary proteins, in addition to Leishmania antigens ${ }^{(3)}$. Another aspect discussed in the review by Duarte et al was the role of adjuvants, which also are important in inducing a Th1 immune response. There have been several adjuvants tested including recombinant interleukin-12 (IL-12), Bacillus Calmette-Guérin (BCG), monophosphoryl A, cytosine-guanine repeats $(\mathrm{CpG})$, recombinant virus and others. Activation of innate immunity is critical for mounting the proper response, via Toll-like receptors, that results in induction of acquired immunity and protection ${ }^{(4)}$.

Another important issue discussed in this review is the cost of vaccine development and implementation, which need to be taken into account ${ }^{(4)}$ in terms of public health guidelines. To date, reservoir and vector control measures have been costly and of relatively low efficacy. It is not clear which population(s) should be the target for vaccination in endemic areas. The excellent review by Duarte et al, covers most of the issues related to development of an effective vaccine for leishmaniasis and the challenges ${ }^{(4)}$. Although, there is still not an effective human vaccine for leishmaniasis, new antigens, adjuvants and improved understanding of the immune factors underlining effective control of parasite replication and pathology give hope for future success.

\section{Acknowledgments}

We thank Patrick Kelly, PhD (University of Iowa) for his review and helpful suggestion to this editorial.

\section{Conflicts of interest}

The authors declare that there are no conflicts of interest.

\section{Financial Support}

This work was supported in part by grants from the US National Institutes of Health (P50 AI-30639) and Conselho Nacional de Desenvolvimento Científico e Tecnológico (CNPq).

\section{REFERENCES}

1. Wilson ME, Jeronimo SM, Pearson RD. Immunopathogenesis of infection with the visceralizing Leishmania species. Microb Pathog 2005; 38:147-160.

2. Blackwell JM, Fakiola M, Ibrahim ME, Jamieson SE, Jeronimo SB, Miller EN, et al. Genetics and visceral leishmaniasis: of mice and man. Parasite Immunol 2009; 31:254-266.

3. Reed SG, Coler RN, Mondal D, Kamhawi S, Valenzuela JG. Leishmania vaccine development: exploiting the host-vectorparasite interface. Expert Rev Vaccines 2016; 15:81-90.

4. Duarte MC, Lage DP, Martins VT, Chavez-Fumigalli MA, Roatt $\mathrm{BM}$, Menezes-Souza D, et al. Recent updates and perspectives on approache for the development of vaccines against visceral leishmaniasis. Rev Soc Bras Med Trop 2016; 49:398-407.

5. Duarte MC, Lage DP, Martins VT, Costa LE, Lage LM, Carvalho $\mathrm{AM}$, et al. A vaccine combining two Leishmania braziliensis proteins offers heterologous protection against Leishmania infantum infection. Mol Immunol 2016; 76:70-79.

6. Scott P, Novais FO. Cutaneous leishmaniasis: immune responses in protection and pathogenesis. Nat Rev Immunol 2016; doi:10.1038/ nri.2016.72.

7. Badaro R, Rocha H, Carvalho EM, Queiroz AC, Jones TC. Leishmania donovani: an opportunistic microbe associated with progressive disease in three immunocompromised patients. Lancet 1986; 1:647-649.

8. Alvar J, Blazquez J, Najera R. Association of visceral leishmaniasis and human immunodeficiency virus infections. J Infect Dis 1989; 160:560-561.

9. Harrison LH, Naidu TG, Drew JS, de Alencar JE, Pearson RD. Reciprocal relationships between undernutrition and the parasitic disease visceral leishmaniasis. Rev Infect Dis 1986; 8:447-453.

10. Fakiola M, Strange A, Cordell HJ, Miller EN, Pirinen M, Su Z, et al. Common variants in the HLA-DRB1-HLA-DQA1 HLA class II region are associated with susceptibility to visceral leishmaniasis. Nat Genet 2013; 45:208-213. 